\title{
Cyanobacteria use both $p$-hydroxybenozate and homogentisate as a precursor of plastoquinone head group
}

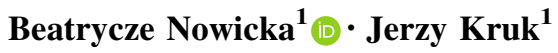

Received: 26 May 2015/Revised: 16 November 2015/Accepted: 7 December 2015/Published online: 29 January 2016

(C) The Author(s) 2016. This article is published with open access at Springerlink.com

\begin{abstract}
Until recently it was believed that cyanobacterial pathway of plastoquinone biosynthesis is analogical to that of higher plants. In plants, homogentisate is a precursor of the hydrophilic head group of plastoquinone. Recent experiments on Synechocystis sp. PCC 6803 have shown that this organism takes advantage of another pathway that resembles ubiquinone biosynthetic pathway of $\alpha$-, $\beta$ - and $\gamma$-proteobacteria. In the present work, we have analysed the content of plastoquinone, tocopherol and tocopherolquinone in six strains of cyanobacteria and compared the obtained results with search for genes of homologues of enzymes participating in tocopherol and ubiquinone biosynthesis. We have shown that inhibition of homogentisate synthesis lowers tocopherol content but does not affect plastoquinone synthesis in Synechococcus sp. PCC 7002. Inhibitors of $p$-hydroxybenzoate and homogentisate prenyltransferases selectively influenced plastoquinone and tocopherol biosynthesis in Synechocystis sp. PCC 6803. Radiolabelled ${ }^{14} \mathrm{C}-p$-hydroxybenzoate was incorporated into plastoquinone by three cyanobacteria species investigated. Although, when ${ }^{14} \mathrm{C}$-homogentisate was added to growth medium, the labelled plastoquinone was found in extracts of the cyanobacteria. Synechocystis sp. PCC 6803 grown in the presence of ${ }^{14} \mathrm{C}$-homogentisate showed also small amounts of the labelled tyrosine, suggesting that cyanobacteria are able to incorporate exogenously added homogentisate into shikimate pathway.
\end{abstract}

Communicated by E. Schleiff.

Beatrycze Nowicka

beatrycze.nowicka@uj.edu.pl; beatrycze.nowicka@wp.pl

1 Department of Plant Physiology and Biochemistry, Faculty of Biochemistry, Biophysics and Biotechnology, Jagiellonian University, Gronostajowa 7, 30-387 Kraków, Poland
Keywords Cyanobacteria $\cdot$ Homogentisate . $p$-Hydroxybenzoate $\cdot$ Plastoquinone $\cdot$ Plastoquinone biosynthesis

\begin{tabular}{ll}
\multicolumn{2}{l}{ Abbreviations } \\
HBA & $p$-Hydroxybenzoate \\
HGA & Homogentisate \\
HPP & 4-Hydroxyphenylpyruvate \\
HPPD & 4-Hydroxyphenylpyruvate dioxygenase \\
HPT & Homogentisate prenyltransferase \\
HBA PRT & $p$-Hydroxybenzoate prenyltransferase \\
ML & Medium light \\
PQ & Plastoquinone \\
PQH & Plastoquinol \\
TC & Tocopherol cyclase \\
$\gamma$-TMT & $\gamma$-Tocopherol methyltransferase \\
Toc & Tocopherol \\
$\alpha$-Toc & $\alpha$-Tocopherol \\
$\gamma$-Toc & $\gamma$-Tocopherol \\
$\alpha$-TQ & $\alpha$-Tocopherolquinone \\
UQ & Ubiquinone
\end{tabular}

\section{Introduction}

Isoprenoid quinones and chromanols are important groups of amphipathic compounds, both containing isoprenoid side-chain and sharing some common steps in their biosynthetic pathways. Among isoprenoid quinones, plastoquinone (PQ), present in all cyanobacteria, participates both in photosynthetic and respiratory electron transport chains (Nowicka and Kruk 2010). In most species of cyanobacteria, PQ is also a cofactor of phytoene 
Fig. 1 Plastoquinone and tocopherol biosynthesis in higher plants. $H G A$ homogentisate, $H P P$ hydroxyphenylpyruvate, $H P P D$ p-hydroxyphenylpyruvate dioxygenase, $H P T$ homogentisate phytyltransferase, $H S T$ homogentisate solanesyl transferase, $P P P$ phytyl diphosphate, $P Q A$ plastoquinone A, $\gamma$-Toc $\gamma$ tocopherol, Tyr tyrosine. Names of the genes are in italics: PDS1 $(H P D)$ gene of $p$ -

hydroxyphenylpyruvate dioxygenase, $P D S 2$ gene of homogentisate solanesyltransferase, VTE1 gene of tocopherol cyclase, VTE2 gene of homogentisate phytyltransferase, VTE3 gene of 2-methyl-6-phytyl benzoquinol methyltransferase<smiles>C=C(O[C@H]1C=C(C(=O)O)C=C[C@H]1O)C(=O)O</smiles><smiles>NC(Cc1ccc(O)cc1)C(=O)O</smiles>

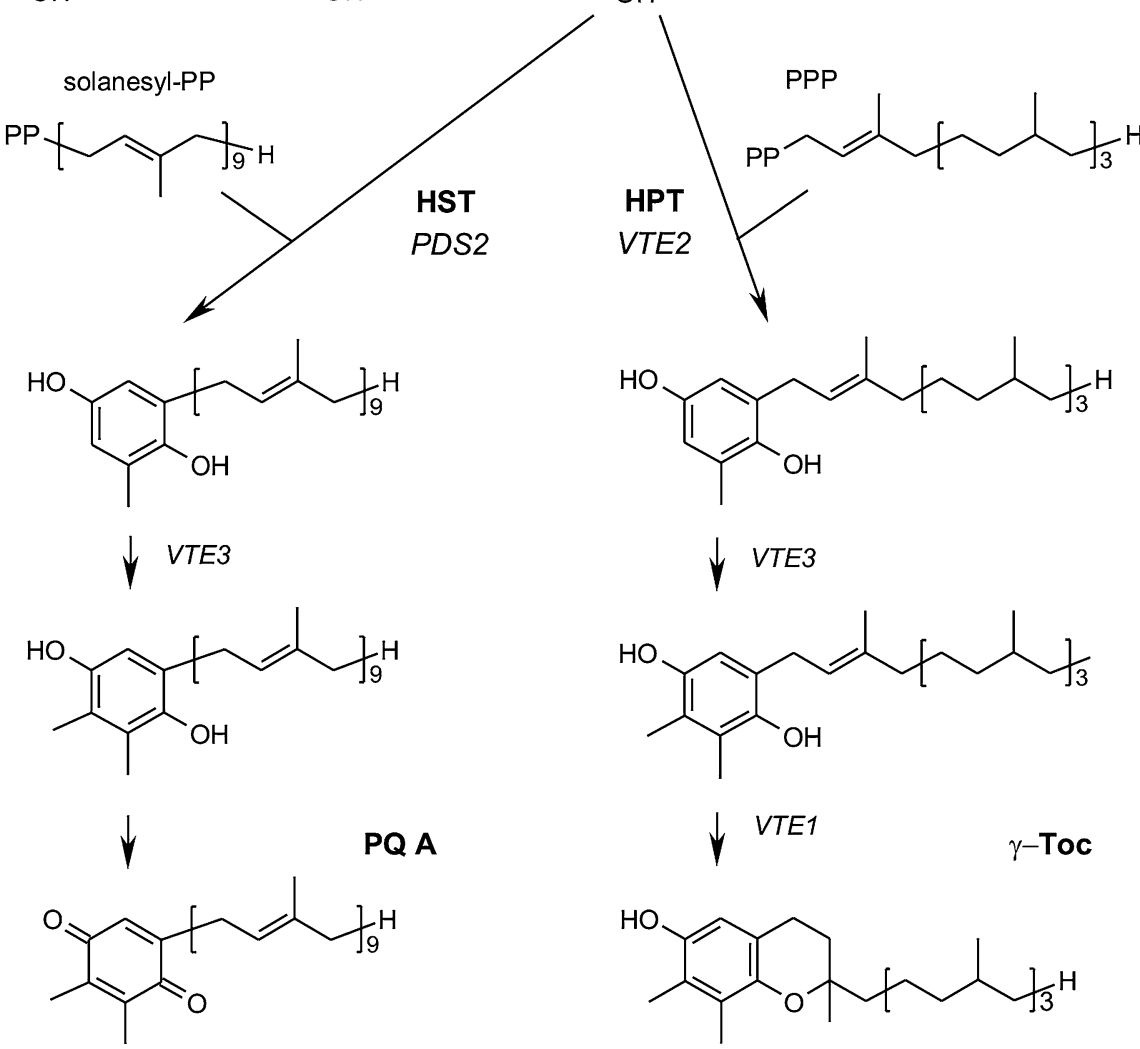

desaturase. This compound also shows antioxidant properties, especially in the reduced form (Nowicka and Kruk 2010). $\alpha$-Tocopherol ( $\alpha$-Toc), a well-known antioxidant, occurs in majority of cyanobacteria, although there are species lacking it, e.g., Synechococcus elongatus sp. PCC 7942 (Munne-Bosch and Alegre 2002). Among oxidation products of $\alpha$-Toc, $\alpha$-tocopherolquinone ( $\alpha$-TQ) can be found in minor amounts in some species of cyanobacteria (Carr and Hallaway 1965).

In higher plants, biosynthetic pathways of PQ and tocopherols (Tocs) are well known. Both pathways share first steps, as both use homogentisate (HGA) as a precursor of hydrophilic head groups of PQ and Toc (Fig. 1) (MèneSaffrané and DellaPenna 2010). Experiments performed on Synechocystis sp. PCC 6803 showed that biosynthetic pathway of $\alpha$-Toc in cyanobacteria is analogical to that of higher plants (DellaPenna 2005). What is more, synthesis of phylloquinone, a cofactor of photosystem I, is very similar in cyanobacteria and plants (Gross et al. 2006). In experiments carried out by Whistance and Threlfall, where radiolabelled HGA was added to the growth medium of Synechococcus elongatus, the presence of labelled PQ in 

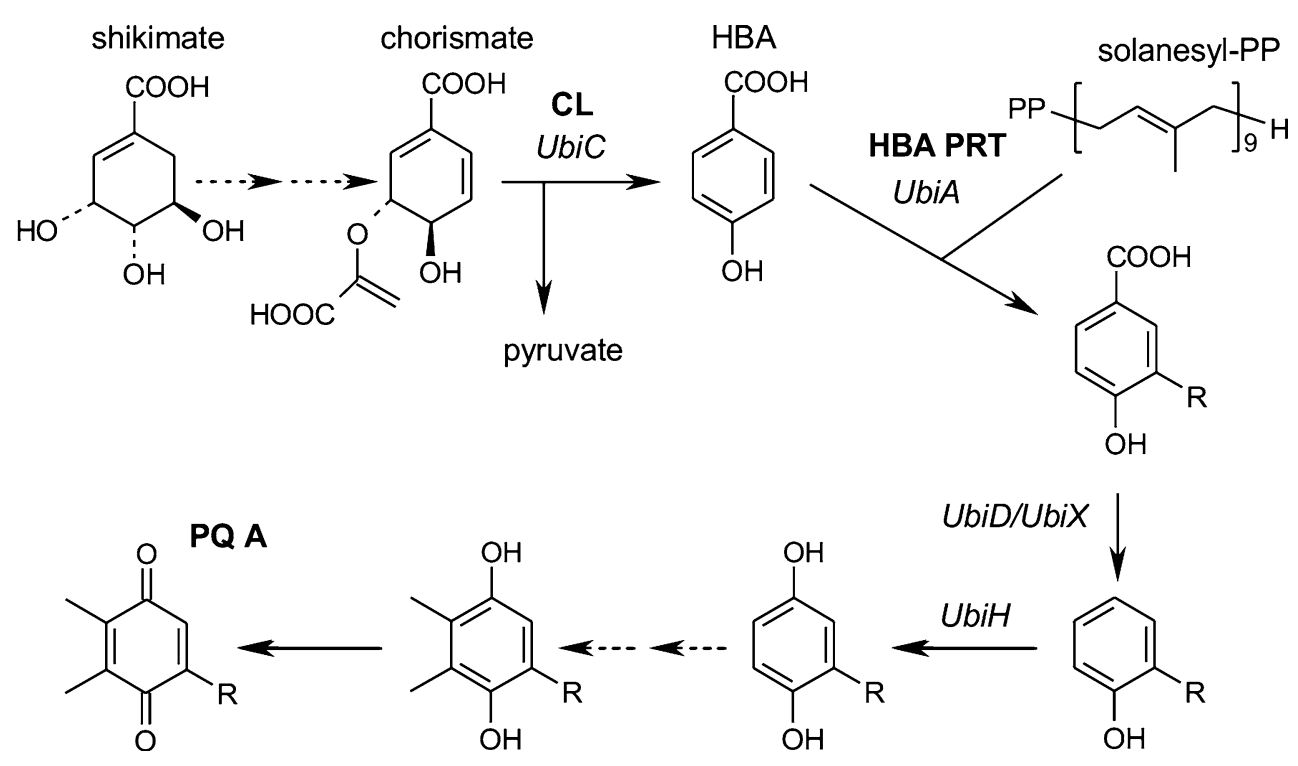

Fig. 2 Plastoquinone biosynthetic pathway in cyanobacteria proposed by (Sakuragi and Bryant 2006). $C L$ chorismate lyase, $H B A$ $p$-hydroxybenzoate, $H B A P R T$-hydroxybenzoate prenyltransferase, $P Q$ A plastoquinone A, $R$ solanesyl side-chain. Names of the genes are in italics: UbiA gene of $p$-hydroxybenzoate prenyltransferase,

extracts from cyanobacteria was observed (Whistance and Threlfall 1970). Therefore, it was assumed that cyanobacteria synthesize PQ via the same pathway as plants.

First data challenging this hypothesis came from experiments on mutants lacking 4-hydroxyphenylpyruvate dioxygenase (HPPD). Arabidopsis thaliana pds1 mutant is deficient both in PQ and Tocs, therefore, it is lethal at early stage of seedling development (Norris et al. 1995). The HPPD mutant of Synechocystis sp. PCC 6803 lacks only $\alpha$ Toc, while its PQ level remains unchanged as compared to the wild type; cyanobacterium Synechococcus elongatus lacks $\alpha$-Toc but synthesizes PQ (Henninger et al. 1965; Dasilva and Jensen 1971; Dahnhardt et al. 2002). On the basis of sequence analysis, which has shown that homologues of genes encoding five enzymes participating in ubiquinone-8 (UQ-8) biosynthesis in E. coli (UbiA, UbiD, UbiE, UbiH, UbiX) are present in 14 cyanobacterial genomes, Sakuragi and Bryant proposed that PQ biosynthetic pathway in cyanobacteria resembles that of UQ in $\alpha-, \beta$ and $\gamma$-proteobacteria (Fig. 2) (Sakuragi and Bryant 2006).

For elucidation of the PQ biosynthetic pathway in cyanobacteria, experiments on deletion mutants were performed. Synechocystis sp. PCC 6803 mutants lacking UbiA homologue were not viable (Schultze et al. 2009). As PQ is the only photosynthetic and respiratory quinone electron carrier in cyanobacteria, this compound is indispensable for metabolism of the cell. To overcome this obstacle, Sadre et al. (2012) used a system with heterologous expression of examined cyanobacterial genes in E. coli mutant strains
UbiC gene of chorismate lyase, UbiD gene of 3-octaprenyl-4hydroxybenzoate decarboxylase, $\mathrm{UbiH}$ gene of 2-octaprenyl-6methoxyphenol hydroxylase, UbiX gene of 3-octaprenyl-4-hydroxybenzoate carboxy-lyase

lacking ubiquinone. As E. coli takes advantage of both UQ8 and menaquinone-8 in its respiratory electron transfer chain, mutants lacking UQ are able to grow. Expression of cyanobacterial homologue of proteobacterial $p$-hydroxybenzoate prenyltransferase (HBA PRT), encoded by Slr0926, in E. coli mutants lacking UbiA, restored UQ-8 biosynthesis. Moreover, it was shown that ${ }^{14} \mathrm{C}$-labelled HBA was used for PQ biosynthesis in Synechocystis sp. PCC 6803 (Sadre et al. 2012). Sadre et al. also examined properties of Slr0926 product isolated from transgenic E. coli and proved that the transgenic protein indeed was HBA prenyltransferase (Sadre et al. 2012). Using an analogical approach, the same group confirmed that cyanobacterial homologues of UbiC, UbiD and UbiX, encoding chorismate lyase and two 4-hydroxy-3-solanesylbenzoate decarboxylases, respectively, participate in PQ biosynthesis (Pfaff et al. 2014).

The aim of the present experiments was to broaden our knowledge concerning PQ biosynthesis in cyanobacteria and to elucidate discrepancies between results of early and recent research on this topic. As in early research carried out by Whistance and Threlfall (Whistance and Threlfall 1970), radiolabelled HGA was incorporated into PQ, whereas in latest research (Sadre et al. 2012; Pfaff et al. 2014), the pathway analogical to UQ biosynthetic pathway present in proteobacteria, in which $p$-hydroxybenzoate (HBA) serves as a precursor of PQ head group was proved to occur in Synechocystis sp. PCC 6803, there is a need to explain these differences. The other interesting question is 
if the pathway of PQ biosynthesis found in model cyanobacterium Synechocystis sp. PCC 6803 is common in other cyanobacterial species.

The obtained results indicate that the pathway of PQ biosynthesis proposed by Sakuragi and Bryant occurs also in cyanobacteria species other than Synechocystis sp. PCC 6803. We also provide data concerning PQ, $\alpha$-Toc and $\alpha$ TQ content in selected strains of cyanobacteria. The results of experiments with inhibitors of $\alpha$-Toc and UQ biosynthesis also confirm the UQ-like pathway of PQ biosynthesis of cyanobacteria. Finally, we show that exogenously applied ${ }^{14} \mathrm{C}-\mathrm{HGA}$ indeed can be used for PQ biosynthesis, besides HBA. This effect is probably a result of incorporation of HGA into shikimate pathway.

\section{Materials and methods}

\section{Sequence analysis}

Sequence analysis was performed using BLAST ver. 2.2.25+ (http://www.ncbi.nlm.nih.gov/blast/Blast.cgi) with default settings for protein blast (Altschul et al. 1997, 2005). The search was performed for homologues of ten enzymes participating in $\alpha$-Toc or UQ biosynthesis in genomes of Synechocystis sp. PCC 6803, Synechococcus elongatus (PCC 7942 and PCC 6301), Synechococcus sp. PCC 7002 and Thermosynechococcus elongatus BP-1. The following sequences were used as queries: NP_442493.1 (HPPD from Synechocystis sp. PCC 6803), BAA17774.1 (homogentisate phytyltransferase from Synechocystis sp. PCC 6803), BAK50662.1 (2-methyl-6-phytyl-1,4-benzoquinol methyltransferase from Synechocystis sp. PCC 6803), BAA17775.1 (tocopherol cyclase from Synechocystis sp. PCC 6803), BAK51348.1 ( $\gamma$-tocopherol methyltransferase from Synechocystis sp. PCC 6803), BAE78042.1 (HBA PRT from E. coli), NP_418285.1 (3-octaprenyl-4-hydroxybenzoate decarboxylase, UbiD from $E$. coli), NP_288885.1 (3-octaprenyl-4-hydroxybenzoate carboxy-lyase, UbiX from E. coli), YP_491108.1 (2-octaprenyl-6-methoxyphenol hydroxylase, UbiH from E. coli), YP_491609.1 (bifunctional 2-octaprenyl-6-methoxy-1,4-benzoquinone methylase/S-adenosylmethionine:2-DMK methyltransferase, UbiE from $E$. coli).

\section{Growth conditions}

Cyanobacteria were grown for 6 weeks in sterile liquid media. Synechocystis sp. PCC 6803, Synechococcus elongatus (PCC 7942 and PCC 6301), Thermosynechococcus elongatus BP-1 and Phormidium laminosum were grown in BG-11 medium, while Synechococcus sp. PCC 7002 was grown in medium A for marine cyanobacteria (Stevens et al. 1973). Thermosynechococcus elongatus BP-1 and Phormidium laminosum were grown in a shaker at $45^{\circ} \mathrm{C}$, in low light $\left(10 \mu \mathrm{mol}\right.$ photons $\left./ \mathrm{m}^{2} \mathrm{~s}\right)$. Other strains were grown at $28{ }^{\circ} \mathrm{C}, 2-5 \mu \mathrm{mol}$ photons $/ \mathrm{m}^{2} \mathrm{~s}$.

\section{Prenyllipid extraction and HPLC analysis}

Every sample was obtained from $50 \mathrm{ml}$ of the culture. Cultures were centrifuged $\left(10 \mathrm{~min} \times 5500 \mathrm{~g}, 4{ }^{\circ} \mathrm{C}\right)$ and lyophilized overnight. The obtained lyophilisates (usually $5-15 \mathrm{mg}$ ) were extracted with methanol overnight in a shaker at $4{ }^{\circ} \mathrm{C}$, then re-extracted three times using methanol. Pooled supernatants were evaporated and resuspended in methanol. In the case of $\alpha$-TQ measurements, for $10 \mathrm{mg}$ of lyophilisate used for the extraction, $300 \mu \mathrm{l}$ of final extract was obtained. For $\alpha$-Toc and PQ measurements, final extract was diluted five times. PQ and $\alpha$-Toc were analysed in following RP-HPLC system: $\mathrm{C}_{18}$ column (Nucleosil 100, $250 \times 4 \mathrm{~mm}, 5 \mu \mathrm{m}$, Teknokroma, Spain), methanol:hexane $(340: 20, \mathrm{v} / \mathrm{v})$, flow rate $1.5 \mathrm{ml} / \mathrm{min}$, absorbance detection at $255 \mathrm{~nm}$, fluorescence detection at $\lambda_{\mathrm{ex}}=290 \mathrm{~nm}, \lambda_{\mathrm{em}}=330 \mathrm{~nm}$. For $\alpha-\mathrm{TQH}_{2}$ determination, acetonitrile:methanol:water $(72: 8: 1, v / v)$ was used. For $\alpha$ TQ determination $\mathrm{C}_{18}$ column with platinum post column was used, developed in methanol:water $(95: 5, \mathrm{v} / \mathrm{v})$, at the flow of $1 \mathrm{ml} / \mathrm{min}$ and fluorescence detection as described before (Nowicka and Kruk 2012).

\section{Experiments with inhibitors}

In the experiment concerning response of Synechococcus sp. PCC 7002 to high light, 6-week-old culture was exposed to medium light (ML, $70 \mu \mathrm{mol}$ photons $/ \mathrm{m}^{2} \mathrm{~s}$ ) for $120 \mathrm{~h}$. Samples were taken at $t=0,8,24,48,120 \mathrm{~h}$, extracted and analysed as described before. For $\alpha$-Toc measurements, methanol:water $(99: 1, \mathrm{v} / \mathrm{v})$ and flow rate $1 \mathrm{ml} / \mathrm{min}$ were used.

In the experiment with pyrazolate, Synechococcus sp. PCC 7002 was grown for 5 weeks as described previously with or without $10 \mu \mathrm{M}$ pyrazolate [4-(2,4-dichlorobenzoyl)-1,3-dimethylpyrazol-5-yl toluene-4-sulfonate, Wako Pure Chemicals, Japan]. After 5 weeks, second portion of pyrazolate $(10 \mu \mathrm{l}$ of $50 \mathrm{mM}$ pyrazolate solution for $50 \mathrm{ml}$ of the culture) was added. Then, cultures were exposed to ML. Samples were taken before and after $120 \mathrm{~h}$ of $\mathrm{ML}$ exposure, extracted and analysed using HPLC.

In experiment with inhibitors of HGA- and HBAprenyltransferases, haloxydine (3,5-dichloro-2,6-difluoropyridin-4-ol, Wuhan Yitongtai Biological Co. Ltd., China) and 4-nitrobenzoate (Sigma-Aldrich), respectively, were used. Synechocystis sp. PCC 6803 was grown in BG11 medium. Concentration of the inhibitors was $175 \mu \mathrm{M}$. For all cultures, the same volume of stock culture was used 
as inoculum. After 4 weeks of growth, cyanobacteria were centrifuged $\left(10 \mathrm{~min} \times 5500 \mathrm{~g}, 4^{\circ} \mathrm{C}\right)$. Next, the cells were resuspended in $0.5 \mathrm{ml}$ of distilled water and sonicated three times for $13 \mathrm{~min}, 60 \%$ duty cycle, $35 \%$ of maximal power (Vibra cell, Sonics and Materials Inc.). Between sonication cycles, cultures were frozen in liquid nitrogen and thawed. After sonication, $1.5 \mathrm{ml}$ of chloroform:methanol mixture $(2: 1, \mathrm{v} / \mathrm{v})$ was added to each sample. Samples were then extracted for $2 \mathrm{~h}$ on a vortex in $4{ }^{\circ} \mathrm{C}$ and centrifuged $(10 \mathrm{~min} \times 9000 \mathrm{~g})$. Chloroform phases were pooled, evaporated, dissolved in $200 \mu \mathrm{l}$ of methanol and analysed with HPLC system.

\section{Cultures with radiolabelled precursors}

Radiolabelled precursors, i.e., ${ }^{14} \mathrm{C}-\mathrm{HBA}$ and ${ }^{14} \mathrm{C}$-HGA, were synthesized from ${ }^{14} \mathrm{C}$-Tyr (Hartmann Analytic, Germany, $250 \mu \mathrm{Ci}$ in $2.5 \mathrm{ml}, 485 \mathrm{mCi} / \mathrm{mmol}$ ) as described in Threllfall and Whistance (1971) and Ashby and Edwards (1990). For experiments with labelled precursors, Synechocystis sp. PCC 6803, Synechococcus elongatus PCC 7942 and Synechococcus sp. PCC 7002 were used. In the case of Synechococcus sp. PCC 7002, sterilized solutions of radiolabelled HGA and HBA, mixed with the respective unlabelled compounds, were added to 6-week-old cultures. Then, the cultures were exposed to ML for $120 \mathrm{~h}$. Samples ( $25 \mathrm{ml}$ of each culture) were taken after 48 and $120 \mathrm{~h}$. The whole experiment was repeated and samples were taken after 5, 10, and $24 \mathrm{~h}$. In the case of Synechocystis sp. PCC 6803 and Synechococcus elongatus PCC 7942, radiolabelled compounds were added to 12-dayold cultures. Samples (50 ml of each culture) were taken after 1 and 3 weeks of growth in the presence of labelled compounds. For incorporation experiments, $2.71 \mathrm{ml}$ of the ${ }^{14} \mathrm{C}$ HGA stock solution $(0.92 \mathrm{mM}, \approx 5 \mu \mathrm{Ci} / \mathrm{ml})$ or the corresponding amount of ${ }^{14} \mathrm{C}$-HBA was added to $50 \mathrm{ml}$ of cell culture (final precursor conc. $50 \mu \mathrm{M}$ ).

Samples were centrifuged $\left(15 \mathrm{~min} \times 5500 \mathrm{~g}, 4^{\circ} \mathrm{C}\right)$, the pellet was suspended in $600 \mu \mathrm{l}$ of distilled water, sonicated $(2 \times 15 \mathrm{~min}$ at $70 \%$ duty cycle, Sonicator 4710 , Cole Parmer). Between sonication cycles, samples were frozen in liquid nitrogen and thawed. For Synechococcus elongatus PCC 7942, one sonication for 6 min was used. Next, samples were extracted with chloroform:methanol (2:1, $\mathrm{v} / \mathrm{v}$ ) for $2 \mathrm{~h}$ on a vortex in $4{ }^{\circ} \mathrm{C}$. Extracts were centrifuged $(10 \mathrm{~min} \times 9000 \mathrm{~g})$, then chloroform phases were collected, evaporated, dissolved in $100 \mu \mathrm{l}$ of chloroform and spotted on TLC plate with silica gel $(20 \times 20 \mathrm{~cm}$, thickness $0.2 \mathrm{~mm}$, DC-Alufolien, Kieselgel $\mathrm{F}_{254}$, Merck). The plates were developed in chloroform stabilized with amylene (Chempur, Poland), exposed to Kodak phosphor screen for $72 \mathrm{~h}$ and scanned with phosphorimager (BioRad Molecular Imager FX). Cultivation with labelled precursors, extraction and TLC separation was repeated twice for each strain.
Alternatively, $150 \mu \mathrm{l}$ of ${ }^{14} \mathrm{C}$-HGA solution was added to $100 \mathrm{ml}$ of 7-week-old culture of Synechocystis sp. PCC 6803. Fifty $\mathrm{ml}$ of the culture was collected after 17 and $25 \mathrm{~h}$, and extracted as described in the experiment with haloxydine and 4-nitrobenzoate. Hydrophilic phases were collected and concentrated by evaporation. Then, samples were spotted on TLC plate with silica gel. The plates were developed in toluene:acetic acid:water $(25: 25: 1, \mathrm{v} / \mathrm{v})$, then exposed to Kodak phosphor screen for a week and scanned with phosphorimager.

For an experiment with ${ }^{14} \mathrm{C}$-Tyr, the labelled precursor was added to 5-week-old cultures of Synechococcus elongatus PCC 6301 and PCC 7942. Samples $(75 \mathrm{ml}$ of each culture) were taken after $72 \mathrm{~h}$ and extracted as described above. Chloroform phases of the obtained extracts were evaporated, resuspended in a small volume of chloroform and separated using TLC as described above, with chloroform as a mobile phase.

\section{Results}

\section{Comparison of genes encoding enzymes of plant $\alpha$-Toc/PQ biosynthetic pathway and UQ-like pathway with prenyllipid content in selected species of cyanobacteria}

As Synechococcus elongatus (formerly known as Anacystis nidulans) was used in early experiments by Whistance and Threlfall (1970), whereas Synechocystis sp. PCC 6803 was used for experiments concerning $\alpha$-Toc and PQ biosynthesis more recently (Dahnhardt et al. 2002; Sadre et al. 2012; Pfaff et al. 2014), these species were used also in our experiments. Two other species chosen were: Thermosynechococcus elongatus, in which there are no close homologues of genes encoding enzymes of plant $\alpha$-Toc and PQ biosynthetic pathway and Synechococcus sp. PCC 7002 , in which these homologues are present.

Analysis of prenyllipids and bioinformatic analysis of genes encoding enzymes required for PQ and UQ biosynthesis in selected strains of cyanobacteria were performed. The obtained results enabled to correlate the presence of homologues of enzymes engaged in prenyllipid biosynthetic pathways with the occurrence of the prenyllipids analysed.

The results of sequence analysis are shown in Table 1, whereas prenyllipid content is presented in Table 2. It is evident that strains lacking homologues of HPPD, homogentisate phytyltransferase and tocopherol cyclase, are deficient also in $\alpha$-Toc and $\alpha$-TQ. On the other hand, the genes required for the postulated PQ biosynthetic pathway in cyanobacteria are found in all strains. Unfortunately, for Phormidium laminosum, only sequences of 
Table 1 Presence of genes for homologues of enzymes participating in $\alpha$-tocopherol biosynthesis and selected enzymes of ubiquinone biosynthetic pathway of $E$. coli in the examined cyanobacterial strains

\begin{tabular}{|c|c|c|c|c|c|}
\hline \multirow[t]{2}{*}{ Species/strain } & \multicolumn{5}{|c|}{ Enzymes of $\alpha$-Toc biosynthetic pathway } \\
\hline & slr0090 (HPPD) & $\operatorname{slr} 1736(\mathrm{HPT})$ & $\begin{array}{l}\text { sll0418 (MPBQ } \\
\text { MT) }\end{array}$ & slr1737 (TC) & $\operatorname{slr0089}(\gamma-\mathrm{TMT})$ \\
\hline Synechococcus elongatus PCC 6301 & - & - & BAD79117.1 & - & BAD79117.1 \\
\hline Identities/positives & & & $74 / 84 \%$ & & $36 / 52 \%$ \\
\hline Synechococcus elongatus PCC 7942 & - & - & ABB56628.1 & - & ABB56628.1 \\
\hline Identities/positives & & & $74 / 84 \%$ & & $36 / 52 \%$ \\
\hline Synechococcus sp. PCC 7002 & WP_012308304.1 & WP_041443778.1 & WP_012308332.1 & WP_012306299.1 & WP_012307402.1 \\
\hline Identities/positives & $33 / 49 \%$ & $59 / 72 \%$ & $78 / 88 \%$ & $47 / 63 \%$ & $57 / 73 \%$ \\
\hline $\begin{array}{l}\text { Synechocystis sp. PCC } 6803 \text { Genes used } \\
\text { as a query }\end{array}$ & WP_010873791.1 & WP_010872404.1 & WP_010873108.1 & WP_010872405.1 & WP_014407146.1 \\
\hline Thermosynechococcus elongatus $\mathrm{BP}-1$ & - & - & NP_682516.1 & - & NP_682516.1 \\
\hline Identities/positives & & & $76 / 88 \%$ & & $37 / 51 \%$ \\
\hline \multirow[t]{2}{*}{ Species/strain } & \multicolumn{5}{|c|}{ Enzymes of UQ biosynthetic pathway in $E$. coli } \\
\hline & $\begin{array}{l}\text { HBA PRT } \\
\text { (UbiA) }\end{array}$ & UbiD & UbiE & UbiH & UbiX \\
\hline Synechococcus elongatus PCC 6301 & BAD80321.1 & BAD79712.1 & BAD80228.1 & BAD80228.1 & BAD80375.1 \\
\hline Identities/positives & $32 / 53 \%$ & $48 / 64 \%$ & $34 / 53 \%$ & $31 / 50 \%$ & $42 / 58 \%$ \\
\hline Synechococcus elongatus PCC 7942 & ABB57994.1 & ABB58618.1 & ABB58085.1 & ABB58085.1 & ABB57940.1 \\
\hline Identities/positives & $33 / 53 \%$ & $48 / 64 \%$ & $34 / 53 \%$ & $31 / 50 \%$ & $42 / 58 \%$ \\
\hline Synechococcus sp. PCC 7002 & WP_012307014.1 & WP_012306736.1 & WP_012307559.1 & WP_012307559.1 & WP_012307025.1 \\
\hline Identities/positives & $36 / 57 \%$ & $46 / 63 \%$ & $32 / 53 \%$ & $33 / 52 \%$ & $39 / 57 \%$ \\
\hline Synechocystis sp. PCC 6803 & WP_010874074.1 & WP_010871507.1 & WP_010871480.1 & WP_010871480.1 & WP_010871388.1 \\
\hline Identities/positives & $34 / 54 \%$ & $45 / 62 \%$ & $31 / 51 \%$ & $32 / 47 \%$ & $42 / 59 \%$ \\
\hline Thermosynechococcus elongatus BP-1 & NP_681192.1 & NP_683015.1 & NP_683079.1 & NP_683079.1 & NP_681822.1 \\
\hline Identities/positives & $34 / 56 \%$ & $45 / 62 \%$ & $40 / 57 \%$ & $32 / 46 \%$ & $43 / 60 \%$ \\
\hline
\end{tabular}

$H B A$ PRT 4-hydroxybenzoate prenyltransferase, $H P P D$ 4-hydroxyphenylpyruvate dioxygenase, $H P T$ homogentisate phytyltransferase, $M P B Q$ $M T$ 2-methyl-6-phytyl-1,4-benzoquinol methyltransferase, TC tocopherol cyclase, $\gamma$-TMT $\gamma$-tocopherol methyltransferase, $\alpha$-Toc $\alpha$-tocopherol, UbiD 3-octaprenyl-4-hydroxybenzoate decarboxylase, $U b i E$ bifunctional 2-octaprenyl-6-methoxy-1,4-benzoquinone methylase/S-adenosylmethionine:2-DMK methyltransferase, UbiH 2-octaprenyl-6-methoxyphenol hydroxylase, UbiX 3-octaprenyl-4-hydroxybenzoate carboxy-lyase, $U Q$ ubiquinone

Table 2 Composition of isoprenoid lipids in the examined cyanobacterial strains

\begin{tabular}{llll}
\hline Species/strain & $\mathrm{PQ}_{\text {tot }}(\mu \mathrm{mol} / \mathrm{g} \mathrm{DW})$ & $\alpha-\mathrm{Toc}(\mu \mathrm{mol} / \mathrm{g} \mathrm{DW})$ & $\alpha-\mathrm{TQ}(\mathrm{nmol} / \mathrm{g} \mathrm{DW})$ \\
\hline $\begin{array}{l}\text { Phormidium laminosum } \\
\text { Synechococcus elongatus }\end{array}$ & $0.65 \pm 0.05$ & $0.369 \pm 0.023$ & $3.9 \pm 0.3$ \\
PCC 6301 & $0.61 \pm 0.08$ & - & - \\
PCC 7942 & $0.59 \pm 0.03$ & - & - \\
Synechococcus sp. PCC 7002 & $0.57 \pm 0.05$ & $0.012 \pm 0.001$ & $-{ }^{\mathrm{a}}$ \\
Synechocystis sp. PCC 6803 & $0.70 \pm 0.05$ & $0.198 \pm 0.004$ & $12.1 \pm 3.4$ \\
Thermosynechococcus elongatus BP-1 & $3.63 \pm 0.56$ & - & - \\
\hline
\end{tabular}

The procedure used, resulted in oxidation of quinols to quinones, so only total content of both of these forms was determined

$D W$ dry weight, $P Q_{t o t}$ total plastoquinone, $\alpha$-Toc $\alpha$-tocopherol, $\alpha$-TQ $\alpha$-tocopherolquinone

The data are mean \pm SE, $n=3-10$

${ }^{a}$ Results of other experiments have shown that in high-light stressed cyanobacteria, trace amounts of $\alpha$-TQ were observed 
selected genes are known presently. Although, the prenyllipid content of that cyanobacterium was included because it is interesting as $P$. laminosum, similar to $T$. elongatus, is a thermophilic species (see "Discussion" section).

Interestingly, PQ content was similar in all the strains, except for T. elongatus BP-1, where it was about six times higher (Table 2). $\alpha$-Toc and $\alpha$-TQ were present only in some species. The level of $\alpha$-Toc varied among the investigated species. The highest content of $\alpha$-Toc was found in thermophilic P. laminosum (Table 2). In Synechococcus sp. PCC 7002, high variations in the content of $\alpha$-Toc were observed. In most of the cultures (9), its level was low, but there were some cultures (3) where only trace amounts of $\alpha$-Toc were detected, whereas in three other cultures, high level of $\alpha$-Toc was found (up to $0.463 \mu \mathrm{mol} /$ g DW). Very low content of $\alpha$-TQ was observed only in species having $\alpha$-Toc (Table 2).

\section{Experiments with selective inhibitors of enzymes participating in plant $\alpha$-Toc/PQ biosynthetic pathway and UQ biosynthetic pathway in proteobacteria}

Pyrazolate is an inhibitor of HPPD, a key enzyme participating in $\alpha$-Toc and PQ biosynthesis in plants. Preliminary experiments, where inhibitor was added during inoculation of the cultures, which were then grown for 4 weeks in low light, showed that there were no differences in prenyllipid content as compared to the control culture (data not shown). Either inhibitor was degraded or, as inhibition was only partial, when growth was slow, $\alpha$-Toc accumulated over time. However, in other preliminary experiments, we have discovered that Synechococcus sp. PCC 7002 was able to acclimate to light of medium intensity (ML, 70 $\mu \mathrm{mol}$ photons $/ \mathrm{m}^{2} \mathrm{~s}$ ), which was accompanied by an increase in $\alpha$-Toc level during $120 \mathrm{~h}$ of ML treatment (data not shown). During such fast response, the effect of pyrazolate should be visible. Therefore, the impact of pyrazolate on ML-induced $\alpha$-Toc increase in Synechococcus sp. PCC 7002 was investigated. The inhibitor was added to 5-week-old culture of Synechococcus sp. PCC 7002 and then the culture was exposed to ML. The increase in $\alpha$-Toc content in response to ML treatment was partially inhibited in the presence of pyrazolate, whereas there was no difference in the case of the change of $\mathrm{PQ}$ content when compared to the control (Fig. 3).

Impact of inhibitors of prenyltransferases participating in quinone biosynthesis on the content of $\alpha$-Toc and PQ was also examined in model cyanobacterium Synechocystis

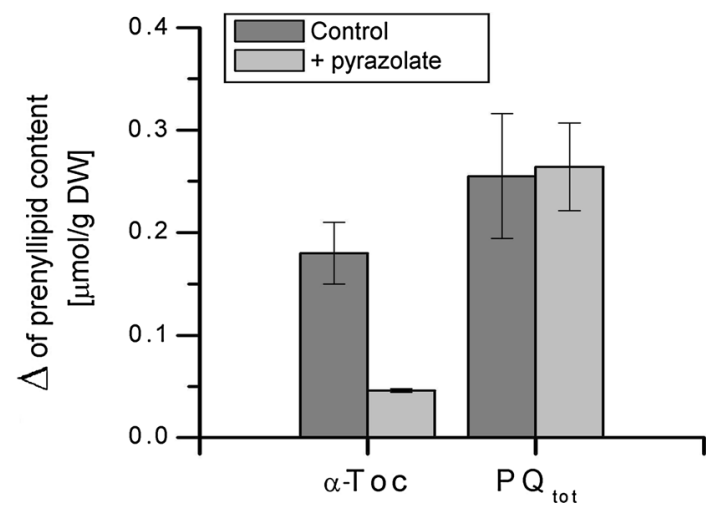

Fig. $3 \alpha$-Tocopherol and plastoquinone content in Synechococcus sp. PCC 7002: control and culture with pyrazolate, during $120 \mathrm{~h}$ of exposure to medium light ( $70 \mu \mathrm{mols}$ photons $\left./ \mathrm{m}^{2} \mathrm{~s}, \mathrm{ML}\right)$ expressed as a difference between prenyllipid content after and before ML treatment. $\alpha$-Toc, $\alpha$-tocopherol; $\mathrm{PQ}_{\text {tot }}$, total plastoquinone. The data are mean $\pm \mathrm{SE}$

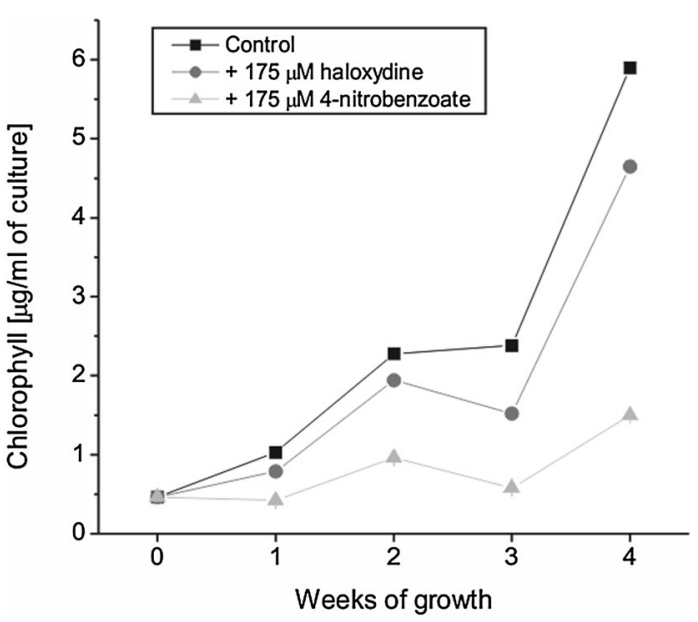

Fig. 4 Chlorophyll a content in cultures of Synechocystis sp. PCC 6803 grown on the medium with inhibitors of plant $\alpha$-Toc/PQ biosynthetic pathway (haloxydine) and UQ biosynthesis (4nitrobenzoate)

sp. PCC 6803. Haloxydine is an inhibitor of HGA prenyltransferases, participating in PQ and $\alpha$-Toc biosynthesis in higher plants, whereas 4-nitrobenzoate is an inhibitor of HBA prenyltransferase engaged in UQ biosynthesis (Sadre et al. 2010; Quinzii et al. 2012). Addition of 4-nitrobenzoate significantly slowed down the culture growth, monitored as chlorophyll content in the liquid culture (Fig. 4). In cultures with haloxydine, content of $\alpha$-Toc was lower than in the control, whereas PQ level remained unchanged. In the culture with 4-nitrobenzoate, PQ content was lower than in the control, whereas $\alpha$-Toc level increased (Table 3). 
Table 3 The effect of inhibitors of plant $\alpha$-Toc/PQ biosynthetic pathway (haloxydine) and UQ biosynthesis (4-nitrobenzoate) on $\alpha$-tocopherol and plastoquinone content in Synechocystis sp. PCC 6803 cultures

\begin{tabular}{lllr}
\hline & \multicolumn{2}{l}{ Prenyllipid content in 4-week-old cultures (mol/100 mols of Chl) } \\
\cline { 2 - 4 } & Control & $+175 \mu \mathrm{M}$ haloxydine & $+175 \mu \mathrm{M}$ 4-nitrobenzoate \\
\hline $\mathrm{PQ}_{\text {tot }}$ & $1.77 \pm 0.03$ & $1.90 \pm 0.11$ & $0.25 \pm 0.03$ \\
$\alpha$-Toc & $0.27 \pm 0.02$ & 0 & $0.61 \pm 0.01$ \\
\hline
\end{tabular}

The data are mean $\pm \mathrm{SE}$

$P Q_{\text {tot }}$ total plastoquinone, $\alpha$-Toc $\alpha$-tocopherol

\section{Experiments with radiolabelled precursors known to be the intermediates of $P Q$ biosynthesis in plants and UQ biosynthesis in proteobacteria}

Addition of radiolabelled precursors to the cultures resulted in incorporation of both ${ }^{14} \mathrm{C}-\mathrm{HBA}$ and ${ }^{14} \mathrm{C}$-HGA predominantly into PQ (Figs. 5, 6). In 6-week-old culture of Synechococcus sp. PCC 7002 exposed to ML for $120 \mathrm{~h}$ after addition of precursors, detectable amounts of labelled PQ were found after $24 \mathrm{~h}$. There was also another product with high $R_{\mathrm{f}}$ for all samples observed, except for those with

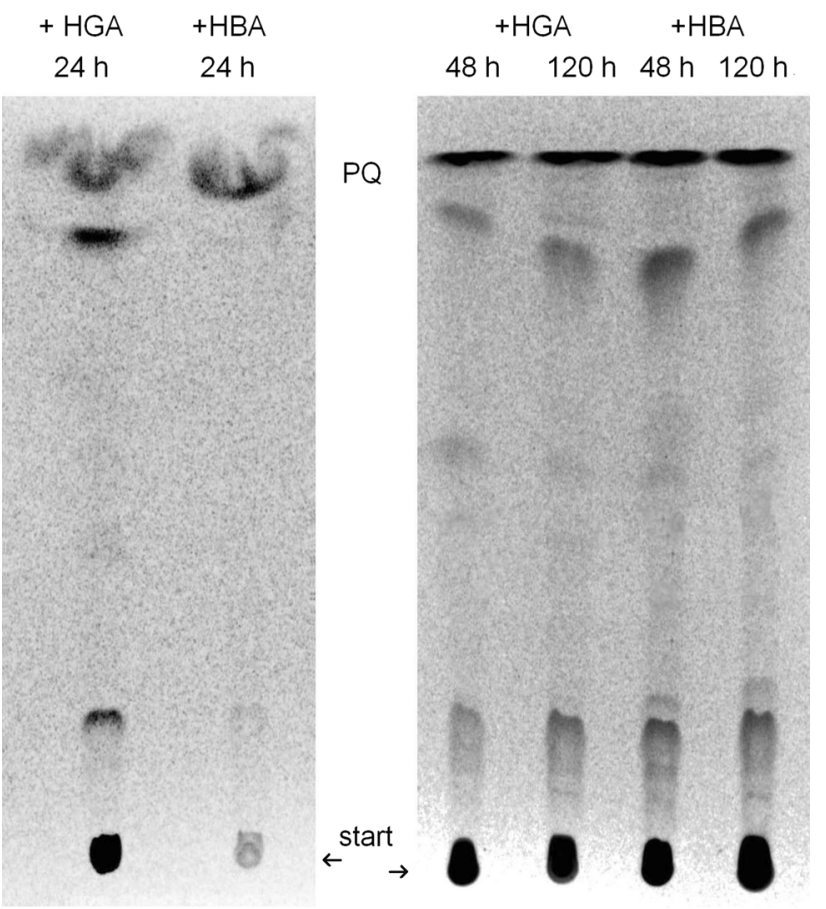

Fig. 5 Autoradiogram of TLC plates of extracts from Synechococcus sp. PCC 7002 exposed to ML. In the first experiment, samples were collected after 48 and $120 \mathrm{~h}$. In the second experiment, samples were collected after $24 \mathrm{~h}$. In the third, after 5 and $10 \mathrm{~h}$, although labelled compounds were present only in trace, barely detectable amounts (data not shown). TLC plates were developed in chloroform. In this mobile phase, HGA and HBA do not move from the start point. $H B A$ $p$-hydroxybenzoate, $H G A$ homogentisate, $P Q$ plastoquinone

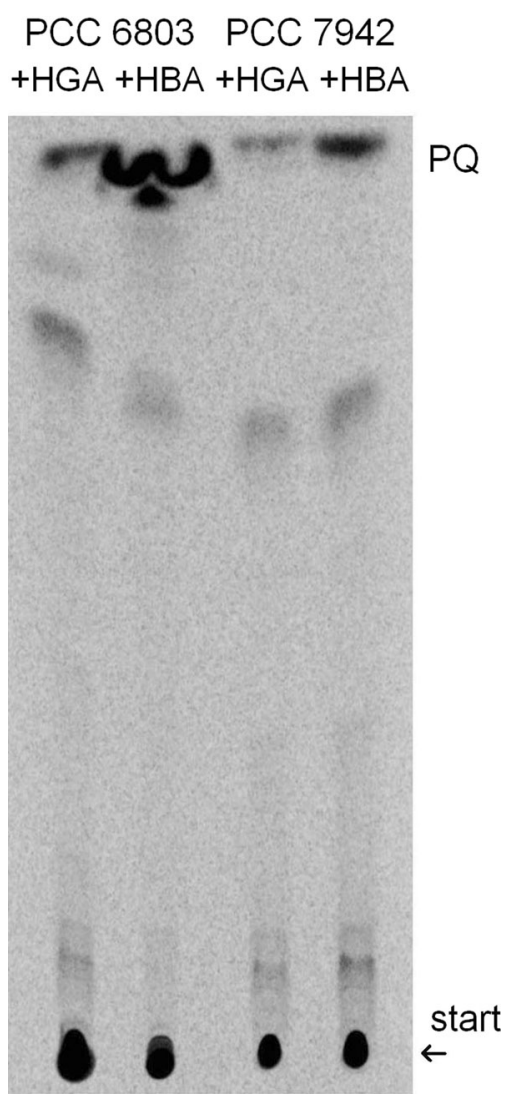

Fig. 6 Autoradiogram of TLC plate of extracts from Synechocystis sp. PCC 6803 and Synechococcus elongatus PCC 7942 grown in low light for 1 week after addition of the labelled precursors to 12-day-old culture. TLC plates were developed in chloroform. HBA $p$-hydroxybenzoate, $H G A$ homogentisate, $P Q$ plastoquinone

${ }^{14} \mathrm{C}-\mathrm{HBA}$ collected after $24 \mathrm{~h}$. In the case of Synechocystis sp. PCC 6803 and Synechococcus elongatus PCC 7942, ${ }^{14} \mathrm{C}-\mathrm{HBA}$ seemed to be incorporated more efficiently than ${ }^{14} \mathrm{C}$-HGA and 3 weeks of growth with precursors turned out to be too long; the labelled compounds were degraded (data not shown).

Analysis of hydrophilic compounds in the extract of Synechocystis sp. PCC 6803, 17 and $25 \mathrm{~h}$ after addition of ${ }^{14} \mathrm{C}$ labelled HGA, showed the presence of labelled Tyr (Fig. 7). The amount of labelled compounds increased with time. 


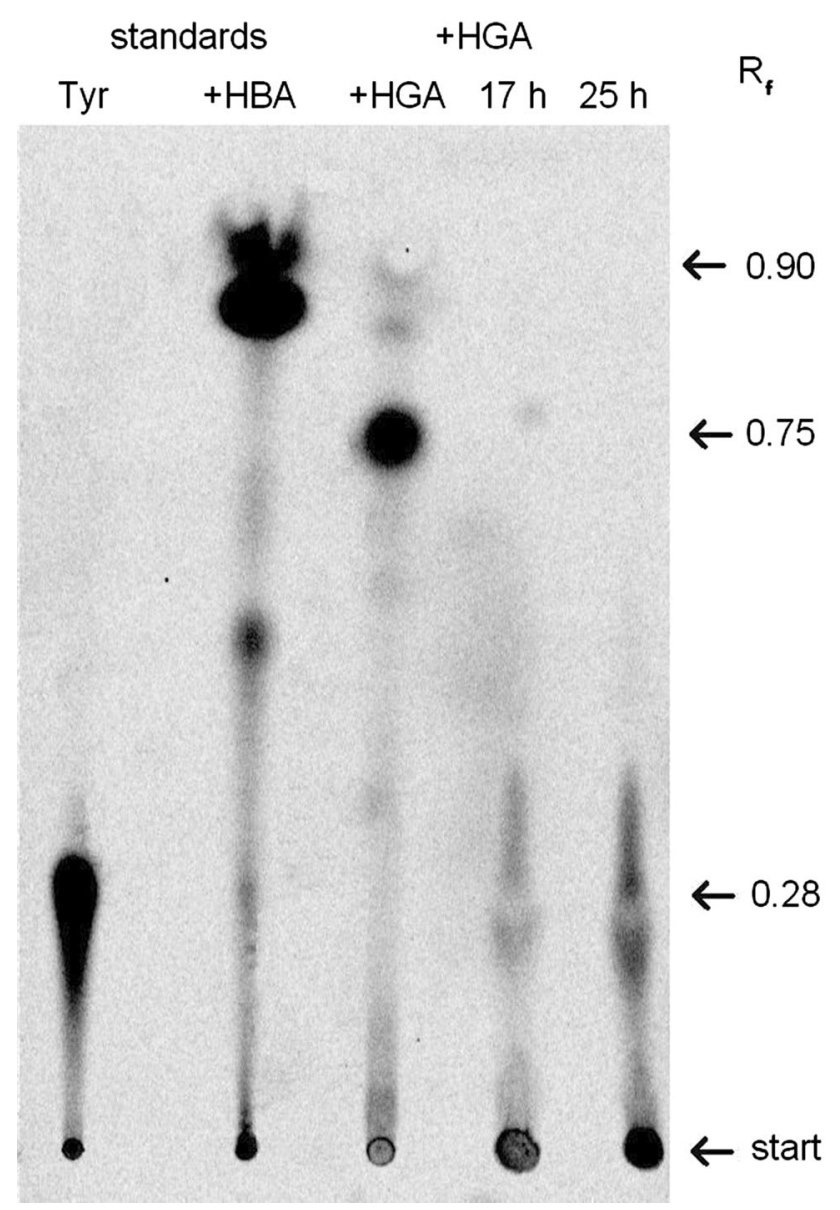

Fig. 7 Autoradiogram of TLC plate of extracts from Synechocystis sp. PCC 6803 after 17 and $25 \mathrm{~h}$ since the addition of ${ }^{14} \mathrm{C}$-HGA. TLC plates were developed in toluene:acetic acid:water $(25: 25: 1, \mathrm{v} / \mathrm{v})$. $H B A$ p-hydroxybenzoate, $H G A$ homogentisate, Tyr tyrosine

In the last experiment, ${ }^{14} \mathrm{C}$-Tyr was added to the cultures of two strains of Synechococcus elongatus, a cyanobacterium that does not have genes encoding HPPD homologues. Therefore, it is not able to convert Tyr into HGA via well known degradation pathway of Tyr, where HPPD performs the most important irreversible step (see Fig. 1). The incorporation of labelled aromatic ring into PQ has been observed, although less evident than in the case of ${ }^{14} \mathrm{C}-\mathrm{HBA}$ and ${ }^{14} \mathrm{C}-\mathrm{HGA}$ (Fig. 8).

\section{Discussion}

In the present experiments, evidence was provided that cyanobacterial strains other than Synechocystis sp. PCC 6803 also take advantage of PQ biosynthetic pathway that is different than that of $\alpha$-Toc, but similar to UQ biosynthesis in proteobacteria. There is a correlation between the presence of HPPD, HPT and TC homologues in strains of cyanobacteria and the presence of $\alpha$-Toc and $\alpha$-TQ.

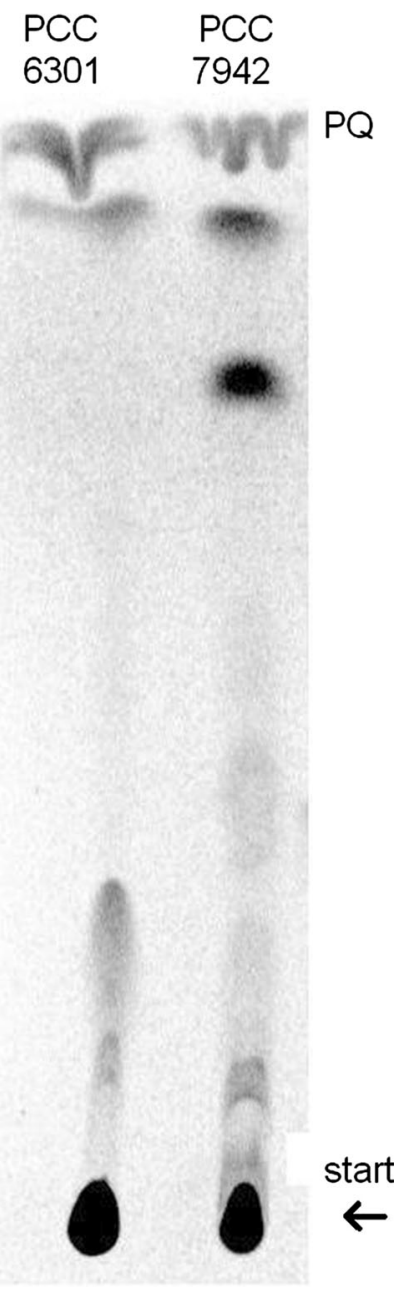

Fig. 8 Autoradiogram of TLC plate of extracts from Synechococcus elongatus PCC 6301 and PCC 7942 after $72 \mathrm{~h}$ since the addition of ${ }^{14} \mathrm{C}$-Tyr. TLC plates were developed in chloroform. $P Q$ plastoquinone

Increased levels of prenyllipids ( $\alpha$-Toc in $P$. laminosum, $\mathrm{PQ}$ in $T$. elongatus) were observed in thermophilic cyanobacteria, which might result from adaptation to the higher temperature of growth, as the increase in $\alpha$-Toc level during heat stress was observed in plants (Bergmüller et al. 2003). It is known that $\alpha$-Toc influences properties of the cell membranes. It was shown in experiments on liposomes that $\alpha$-Toc present in model membranes in certain proportion to other lipids increase the stability of the membrane (Hincha 2008). The absence of HPPD homologues in Synechococcus elongatus PCC 7942 and PCC 6301, as well as in Thermosynechococcus elongatus BP-1, has no impact on PQ synthesis in these strains.

Inhibition of HPPD synthesis in Synechococcus sp. PCC 7002 had no effect on the increase in PQ content in response to medium light treatment, whereas the increase in $\alpha$-Toc level was lowered by about $70 \%$ in the presence of pyrazolate. This effect is analogical to the results obtained by Dahnhardt et al. 
in the experiment with Synechococcus sp. PCC 6803, where mutant with disrupted open reading frame $\Delta s l r 0090$, encoding homologue of plant HPPD, had the same PQ level as the wild type, but was lacking $\alpha$-Toc (Dahnhardt et al. 2002). Results obtained for haloxydine and 4-nitrobenzoate clearly support hypothesis that cyanobacteria use UQ-like pathway for PQ biosynthesis, whereas plant pathway is not branched and used only for $\alpha$-Toc biosynthesis. Inhibition of the prenylation of HBA led to specific inhibition of PQ biosynthesis, whereas inhibition of the prenylation of HGA led to specific inhibition of $\alpha$-Toc biosynthesis. The increased $\alpha$-Toc content in 4-nitrobenzoate treated culture might be an effect of accumulation of shikimate pathway intermediates resulting from inhibition of PQ biosynthesis.

We observed incorporation of labelled HBA into PQ not only in the case of Synechocystis sp. PCC 6803, but also for Synechococcus elongatus PCC 7942 and Synechococcus sp. PCC 7002.

Incorporation of ${ }^{14} \mathrm{C}$-HGA into $\mathrm{PQ}$ was observed in all strains under study. This was also described by Whistance and Threlfall, who observed that the labelled HGA was incorporated into PQ in Synechococcus elongatus (Whistance and Threlfall 1970). Interestingly, the ability to utilize exogenous HGA was found not only for strains able to synthesize this compound (Synechocystis sp. PCC 6803, Synechococcus sp. PCC 7002) but also for strains lacking HPPD homologue, therefore unable to synthesize HGA (Synechococcus elongatus PCC 7942).

During degradation pathways of aromatic compounds, such as HGA, Tyr or Phe, their benzene ring is cleaved, resulting in the formation of carboxylic acids. In the shikimate pathway, where HGA, HBA, Tyr, Phe and other aromatic compounds are formed, substrates for aromatic ring synthesis are sugars (Caspi et al. 2014). If the labelled ring of HGA is cleaved, the probability that degradation products would be introduced into shikimate pathway with high selectivity and efficiency, enabling observation of strong signal due to labelled PQ, is very low. Therefore, it can be concluded that aromatic ring of the applied HGA is not cleaved. Instead, it is introduced into anabolic pathways leading to PQ biosynthesis.

The question is, if HGA can be prenylated by UbiA homologue present in cyanobacteria or if it is converted into other compounds. Sadre and coworkers were not able to observe activity of HGA solanesyltransferase in preparations of recombinant Slr0926, therefore they postulated that HBA prenyltransferase responsible for $\mathrm{PQ}$ biosynthesis is specific for HBA (Sadre et al. 2012). In experiments with haloxydine and 4-nitrobenzoate, we showed that these compounds, known to selectively inhibit prenylation of HGA and HBA, respectively, specifically influence both $\alpha$-Toc and PQ synthesis. Together, these results suggest that $\mathrm{HGA}$ is not prenylated by HBA prenyltransferase of cyanobacteria, but rather converted into other compounds.

Next question is, if a product of HGA conversion can be prenylated in a way that it bypasses prenylation of HBA. Pfaff et al. showed that Synechocystis sp. PCC 6803 knockout mutant of chorismate lyase is barely viable and contains about 30 times less PQ than the wild type (Pfaff et al. 2014). In the experiment with ${ }^{14} \mathrm{C}$-labelled HGA we observed strong signal from the labelled PQ. Therefore, it can be concluded that Synechocystis sp. PCC 6803 does not show any effective alternative PQ biosynthetic pathways, where compounds other than HBA are prenylated to PQ. This means that HGA is converted into a compound that is introduced into the shikimate pathway before the synthesis of HBA.

The presence of labelled Tyr in extracts of Synechocystis sp. PCC 6803 seems to favour this explanation. One can suspect that if HGA is a product of Tyr degradation, there could be a way to reverse this reaction. The conversion of Tyr into $p$-hydroxyphenylpyruvate (HPP) by tyrosine aminotransferase is reversible. However, the second reaction, conversion of HPP into HGA, is a multistep exergonic reaction involving oxidative decarboxylation, phenyl ring hydroxylation and side-chain rearrangement (Borowski et al. 2004). It is therefore irreversible (Rundgren 1983) and simple conversion of HGA into Tyr is rather impossible. However, as Tyr and HBA are both products of the shikimate pathway, sharing common intermediates (see Figs. 1, 2), it seems possible that if a product of HGA conversion is introduced into shikimate pathway, it would result in Tyr production. It is also possible that intermediates of shikimate pathway and of PQ biosynthesis would be metabolized efficiently, while small amounts of Tyr could accumulate.

The hypothetical scheme of HGA conversion is shown in Fig. 9. The proposed intermediates were selected to ensure the least number of unknown steps required to introduce HGA into the shikimate pathway. However, the scheme should be treated only as a proposal to be revised in further research.

In the prokaryotic pathway of HBA synthesis, explored for $E$. coli, this compound is synthesized via the shikimate pathway. The examined eukaryotes, $S$. cerevisiae and mammals can convert Tyr into HBA (Nowicka and Kruk, 2010). In Synechococcus elongatus strains, devoid of an enzyme converting Tyr into HGA, the addition of ${ }^{14} \mathrm{C}$ labelled Tyr to the medium resulted in synthesis of the labelled PQ. It indicates that $S$. elongatus strains have a pathway converting Tyr into HBA, although this question needs further research.

Our results indicate that cyanobacteria have still unknown pathways of conversion of aromatic compounds. These pathways enable both to recycle aromatic 
Fig. 9 Hypothetical scheme of homogentisate conversion into $p$-hydroxybenzoate. Solid lines are reactions known to occur in nature. Dashed lines are hypothetical reactions. $H B A p$ hydroxybenzoate, $H G A$ homogentisate

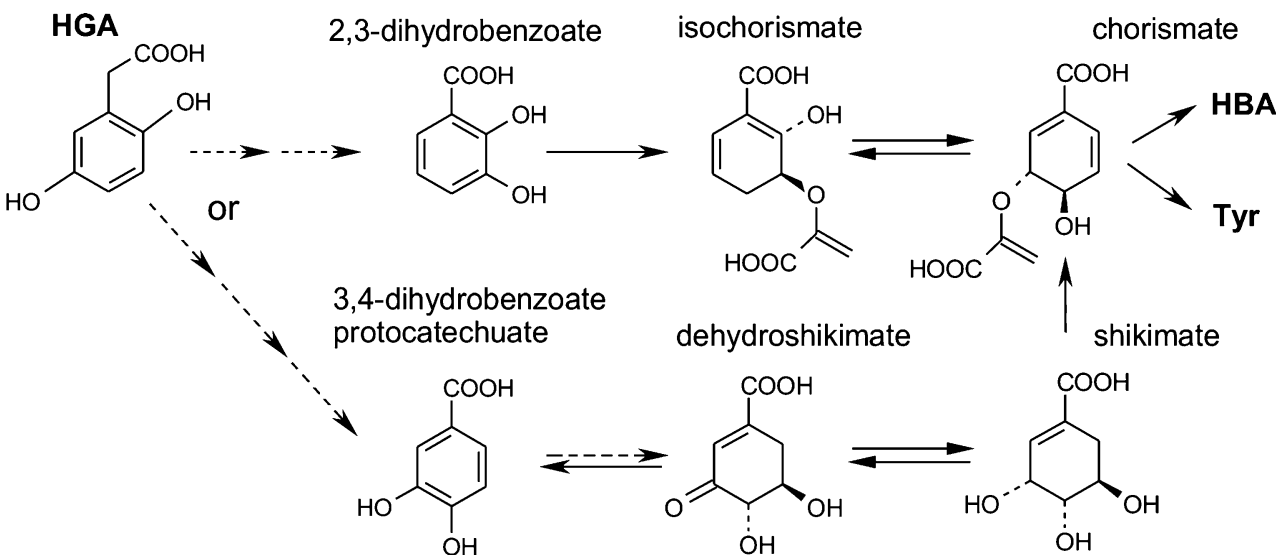

compounds produced in the cell, as well as to take advantage of exogenous compounds.

Author contribution statement J. Kruk is responsible for overall design of the experiment. B. Nowicka optimized the methods used and carried out all experiments and analyses. She has also written the preliminary version of the manuscript. Authors carried out synthesis of radiolabelled homogentisate and $p$-hydroxybenzoate together. They also cooperated in developing and improving of the manuscript.

\section{Compliance with ethical standards}

Financial support This work was supported by a grant from the National Science Center Poland 2013/11/D/NZ1/00303. Scholarship funded by Society-Environment-Technology project co-financed by the European Union, which enabled employment of B. Nowicka on a postdoc position is also acknowledged.

Open Access This article is distributed under the terms of the Creative Commons Attribution 4.0 International License (http://crea tivecommons.org/licenses/by/4.0/), which permits unrestricted use, distribution, and reproduction in any medium, provided you give appropriate credit to the original author(s) and the source, provide a link to the Creative Commons license, and indicate if changes were made.

\section{References}

Altschul SF, Madden TL, Schäffer AA, Zhang J, Zhang Z, Miller W, Lipman DJ (1997) Gapped BLAST and PSI-BLAST: a new generation of protein database search programs. Nucleic Acids Res 25:3389-3402

Altschul SF, Wootton JC, Gertz EM, Agarwala R, Morgulis A, Schäffer AA, Yu YK (2005) Protein database searches using compositionally adjusted substitution matrices. FEBS J 272:5101-5109

Ashby MN, Edwards PA (1990) Elucidation of the deficiency in two yeast coenzyme Q mutants: characterization of the structural gene encoding hexaprenyl pyrophosphate synthetase. J Biol Chem 265:13157-13164
Bergmüller E, Porfirova S, Dörmann P (2003) Characterization of an Arabidopsis mutant deficient in $\gamma$-tocopherol methyltransferase. Plant Mol Biol 52:1181-1190

Borowski T, Bassan A, Siegbahn PEM (2004) 4-Hydroxyphenylpyruvate dioxygenase: a hybrid density functional study of the catalytic reaction mechanism. Biochemistry 43:12331-12342

Carr NG, Hallaway M (1965) Presence of $\alpha$-tocopherolquinone in blue-green algae. Biochem J 97:c9

Caspi R, Altman T, Billington R, Dreher K, Foerster H, Fulcher CA, Holland TA, Keseler IM, Kothari A, Kubo A, Krummenacker M, Latendresse M, Mueller LA, Ong Q, Paley S, Subhraveti P, Weaver DS, Weerasinghe D, Zhang P, Karp PD (2014) The MetaCyc database of metabolic pathways and enzymes and the BioCyc collection of pathway/genome databases. Nucleic Acids Res 42(D1):D459-D471

Dahnhardt D, Falk J, Appel J, van der Kooij TAW, Schulz-Friedrich R, Krupinska K (2002) The hydroxyphenylpyruvate dioxygenase from Synechocystis sp. PCC 6803 is not required for plastoquinone biosynthesis. FEBS Lett 523:177-181

Dasilva EJ, Jensen A (1971) Content of $\alpha$-tocopherol in some bluegreen algae. Biochim Biophys Acta 239:345-347

DellaPenna D (2005) A decade of progress in understanding vitamin E synthesis in plants. J Plant Physiol 162:729-737

Gross J, Cho WK, Lezhneva L, Falk J, Krupinska K, Shinozaki K, Seki M, Herrmann RG, Meurer J (2006) A plant locus essential for phylloquinone (vitamin $\mathrm{K}_{1}$ ) biosynthesis originated from a fusion of four eubacterial genes. $J$ Biol Chem 281:17189-17196

Henninger MD, Bhagavan HN, Crane FL (1965) Comparative studies of plastoquinones. I. Evidence for three quinones in the bluegreen alga Anacystis nidulans. Arch Biochem Biophys 110:69-74

Hincha DK (2008) Effects of $\alpha$-tocopherol (vitamin E) on the stability and lipid dynamics of model membranes mimicking the lipid composition of plant chloroplast membranes. FEBS Lett 582:3687-3692

Mène-Saffrané L, DellaPenna D (2010) Biosynthesis, regulation and functions of tocochromanols in plants. Plant Physiol Biochem 48:301-309

Munne-Bosch S, Alegre L (2002) The function of tocopherols and tocotrienols in plants. Crit Rev Plant Sci 21:31-57

Norris SR, Barrette TR, DellaPenna D (1995) Genetic dissection of carotenoid synthesis in Arabidopsis defines plastoquinone as an essential component of phytoene desaturation. Plant Cell 7:2139-2149

Nowicka B, Kruk J (2010) Occurrence, biosynthesis and function of isoprenoid quinones. Biochim Biophys Acta 1797:1587-1605 
Nowicka B, Kruk J (2012) Plastoquinol is more active than $\alpha$-tocopherol in singlet oxygen scavenging during high light stress of Chlamydomonas reinhardtii. Biochim Biophys Acta 1817:389-394

Pfaff C, Glinedemann N, Gruber J, Frentzen M, Sadre R (2014) Chorismate pyruvate-lyase and 4-hydroxy-3-solanesylbenzoate decarboxylase are required for plastoquinone biosynthesis in the cyanobacterium sp. PCC6803. J Biol Chem 289:2675-2686

Quinzii CM, Tadesse S, Naini A, Hirano M (2012) Effects of inhibiting $\mathrm{CoQ}_{10}$ biosynthesis with 4-nitrobenzoate in human fibroblasts. PLoS One 7:e30606

Rundgren M (1983) Some kinetic properties of 4-hydroxyphenylpyruvate dioxygenase from Pseudomonas sp. strain P.J. 874. Eur J Biochem 133:657-663

Sadre R, Frentzen M, Saeed M, Hawkes T (2010) Catalytic reactions of the homogentisate prenyl transferase involved in plastoquinone-9 biosynthesis. J Biol Chem 285:18191-18198

Sadre R, Pfaff C, Buchkremer S (2012) Plastoquinone-9 biosynthesis in cyanobacteria differs from that in plants and involves a novel 4-hydroxybenzoate solanesyltransferase. Biochem J 442:621-629
Sakuragi Y, Bryant D (2006) Genetic manipulation of quinone biosynthesis in cyanobacteria. In: Golbeck JH (ed) Photosystem I: the light-driven plastocyanin: ferredoxin oxidoreductase. Springer, The Netherlands, pp 205-222

Schultze M, Forberich B, Rexroth S, Dyczmons NG, Roegner M, Appel J (2009) Localization of cytochrome $b_{6} f$ complexes implies an incomplete respiratory chain in cytoplasmic membranes of the cyanobacterium Synechocystis sp. PCC 6803. Biochim Biophys Acta 1787:1479-1485

Stevens SE Jr, Patterson COP, Myers J (1973) The production of hydrogen peroxide by blue-green algae: a survey. J Phycol 9:427-430

Threllfall DR, Whistance GR (1971) Biosynthesis of tocopherols and biogenetically related compounds. Methods Enzymol 18:369-396

Whistance GR, Threlfall DR (1970) Biosynthesis of phytoquinones. Homogentisic acid: a precursor of plastoquinones, tocopherols and alpha-tocopherolquinone in higher plants, green algae and blue-green algae. Biochem J 117:593-600 